\title{
Distribution of Elements Inside Stars
}

\author{
Alecian, G.* \\ LUTH, Observatoire de Paris, PSL, CNRS, Université Paris Diderot, 5 Place Jules Janssen, 92190 Meudon, FRANCE
}

\begin{abstract}
The chemical composition measured in stellar atmospheres is not necessarily the same as in deeper layers (outside the core). Indeed, for a significant fraction of main-sequence G to B types stars the discrepancies between superficial and internal abundances go from a few percent (for the coldest of these stars) to huge factors (for hot chemically peculiar stars). This is due to atomic diffusion process, which may produces elements segregation at some stages of the stellar evolution.
\end{abstract}

Keywords: Stars, Abundances, Atomic diffusion

\section{Introduction}

In standard modelling of stellar envelopes and atmospheres, abundance stratification in mainsequence stars was until recently generally neglected. This was often justified because mixing processes due to turbulence, convection, or large-scale circulation (including mass loss) keep the abundances uniform. Element stratification is due to atomic diffusion (see the pioneering work of Michaud 1970 for Ap stars), and proceeds with very large time scales in stellar interiors, but yet smaller than the structure's evolution time scale. For a normal and cool star like the Sun, the changes of abundances due to atomic diffusion below the outer convection zone, at the present age of the Sun, is estimated to be at most around 10\% (Turcotte et al., 1998), which is very small (but not negligible) in comparison to what is observed for hot chemically peculiar stars (CP stars). However, even such a small effect leads, for instance, to reconsider previous estimates of the age of globular clusters. The abundance stratification build-up in the atmosphere is much faster (from a year to centuries according to the depth in the atmosphere and to the element) and stronger, provided that mixing processes are weak enough. In A and B type main-sequence stars, mixing processes are often weak enough (in particular for slow rotating stars and/or magnetic stars) to allow very effective element separation in upper envelopes and atmospheres. Many stellar types may be concerned by this process, but, here, we consider essentially main-sequence CP stars for which observational effects are striking. A large number of papers about atomic diffusion in stars have been published since 1970, the reader can find a complete presentation and discussion in the recent monograph by Michaud et al. (2015).

The chemically peculiar stars of the main sequence are divided in different groups (AmFm, ApBp, $\mathrm{HgMn}$, etc.), their effective temperatures extend from about $7500 \mathrm{~K}$ to $16000 \mathrm{~K}$, and more if one considers the hotter He-rich stars. About $10-30 \%$ of stars in this temperature range are concerned. Their abundance peculiarities are generally connected to atomic diffusion. Outside this temperature domain, convective motions are too strong for lower temperatures, and stellar wind too strong for higher temperatures to allow element segregation to occur. Actually, because abundance stratification buildup is extremely sensitive to physical conditions, these various groups correspond to various specific physical properties of them (mixing, mass loss, magnetic fields etc.). Figure 1 shows an overview of the peculiarities measured in each group. We may see for instance that in some HgMn stars, $\mathrm{Hg}$ may be overabundant by more a factor of $10^{6}$. Of course, these peculiarities are located in some superficial layers, the total amount of an element inside the star being close to the solar one. When the star evolves and leaves the main-sequence, strong convective motions occur, all the matter is mixed and

*georges,alecian@obspm.fr 


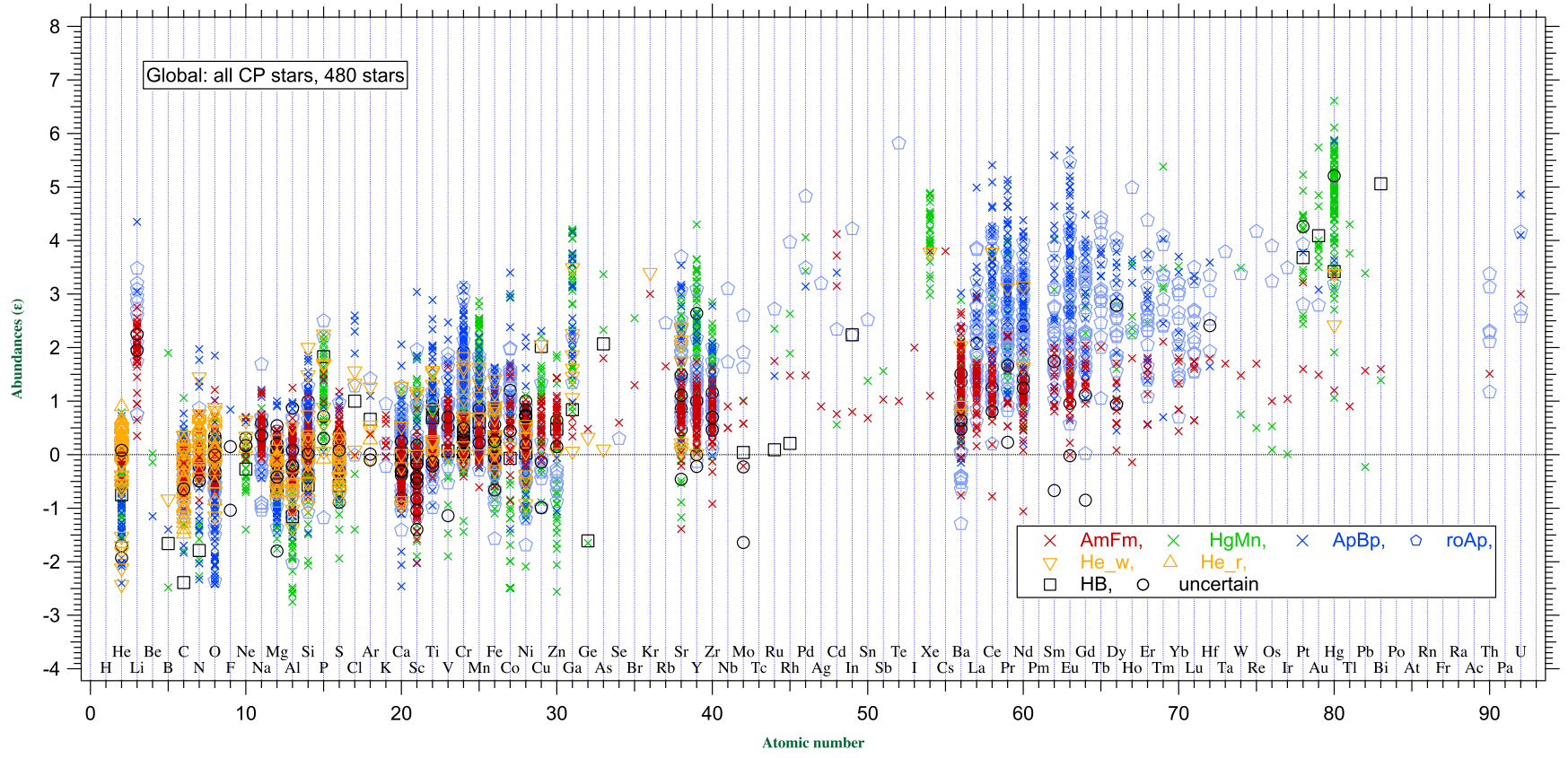

Figure 1. Abundance peculiarities vs. atomic number. Each point is the abundance measured in a CP star for a given element (480 CP stars measured with high resolution spectroscopy are shown here). Abundances $(\epsilon)$ are the logarithm of the abundances divided by the solar ones, the zero line corresponds to solar abundances (from the database of Ghazaryan et al., 2018, Ghazaryan et al., 2019). Notice that most of the dispersion of abundances from star to star are not due to errors of measurements.

no more peculiarities survive. This is why CP stars appear essentially on the main-sequence. Later in the evolution, it happens that mixing processes disappear close enough to the superficial layers, which causes element segregation to proceed again. This is the case for some horizontal branch stars and some white dwarfs.

\section{The atomic diffusion process}

Atomic diffusion is a microscopic process which, inside an anisotropic medium, results in relative average velocities of chemical elements with respect to hydrogen. These velocities are different from one species to the other, and they are generally very small compared to those of large scale motions. Therefore, this physical process that is calculated from first principles, requires a very stable medium to produce observable effects. One knows that this requirement is satisfied in many stars ${ }^{1}$, not only because numerical models including diffusion are the only ones explaining some observed properties of stars, but because abundance stratifications are now well observed (Ryabchikova, 2005, Thiam et al., 2010). Such stratifications imply that the medium is not mixed, and so, atomic diffusion acts necessarily.

\subsection{The diffusion velocity}

The diffusion velocity in the formalism of Chapman \& Cowling (1970) and for plane-parallel geometry may be expressed in simple algebraic form. In general case, it has a vectorial form. The relative velocity of a trace ion (with mass $A_{i} m_{p}$, where $A_{i}$ is the ion atomic mass and $m_{p}$ the proton mass) with respect to protons, in a very schematic and approximate way (only the dominant term is shown), may be written as:

\footnotetext{
${ }^{1}$ Radiative zones are, of course, theoretically predicted since a long time through the various stability criteria depending on local plasma condition, but hydrodynamic instabilities are also caused by rotation. In addition, the stellar mass loss, if strong enough, causes continuous matter flux that impedes abundance stratifications to appear.
} 


$$
V_{D i} \approx D_{i p}\left[A_{i} \frac{m_{p}}{k T}\left(g_{i}^{r a d}-g\right)+\ldots\right],
$$

where $D_{i p}$ is the diffusion coefficient, $g_{i}^{\text {rad }}$ the radiative acceleration, and $g$ gravity. Because each element in a given layer, is generally in several ionization stages, an average diffusion velocity must be computed (weighted by the relative ions population). Notice the minus sign in front of $g$, which expresses the fact that radiative acceleration and gravity act generally in opposite directions. For detailed expression and discussion, see Chapter 2 of Michaud et al. (2015) (see also Alecian, 2014). Radiative acceleration accounts for the momentum transfer from the radiation field to atoms. It is due to absorption of photons by atomic transitions and is specific to each ion. In the absence of photoabsorption, one is left with gravitational settling. Strictly speaking, this only occurs in complete ionisation, very deep in the star, where diffusion time scales are often larger than stellar lifetimes on the main sequence. Radiative acceleration is generally in the range $10^{-2}<g_{i}^{r a d} / g<10^{+3}$ ! So, the sign of the diffusion velocity may be negative ( small $g_{i}^{r a d}$ ) or positive (large $g_{i}^{r a d}$ ). More often, for metals with solar abundance, the modulus of radiative acceleration is larger than gravity. Radiative accelerations should be estimated each time atomic diffusion is suspected to have a significant effect on stellar structure.

\subsection{Radiative acceleration}

The momentum given by the radiation field to particles goes mainly through bound-bound and bound-free interactions of atoms with photons. Because the momentum due to isotropic part of the radiation field cancels, only the net photon flux has to be considered in the computation. Radiative acceleration, in its simplest generic algebraical form may be written as:

$$
g_{\text {rad }}=\frac{1}{m c} \int_{0}^{\infty} \sigma_{\nu} F_{\nu} d \nu
$$

where the net outward radiative energy flux $\left(F_{\nu}\right)$, multiplied by total monochromatic absorption crosssection $\left(\sigma_{\nu}\right)$ for the considered species with mass $m$, is integrated over the radiation frequency. In the case of magnetic atmospheres, due to the polarisation of light, the vectorial form is required. Actually, to compute radiative accelerations is not an easy task. It necessitates taking into account all atomic transitions contributing significantly to the momentum transfer in each stellar layer, for any ion. This implies to consider huge atomic databases and to spend a lot of computer time. We do not go here in the details of this question, the reader can refer to Chapter 3 of Michaud et al. (2015), and many publications starting from Michaud (1970), Watson (1971). Notice also that $F_{\nu}$, for frequencies contributing to the $g_{r a d}$ of a given element, depends on the local abundance of that element (the abundance which is precisely modified by atomic diffusion...).

To illustrate the importance of radiative accelerations, let us consider the case of a main sequence star $\left(T_{\text {eff }}=12000 \mathrm{~K}\right)$. The left panel of Fig. 2 shows the radiative acceleration of iron (solar abundance) and gravity (dashed line). For $\log T \approx 5.5$, the radiative acceleration is almost 10 times larger than gravity. This means that radiative acceleration is able to support up to about 100 times $^{2}$ the solar Fe abundance in these layers. The right panel shows, for the same star, how much the Rosseland averaged opacity increases when iron abundance is multiplied by 10 (dotted line). It appears that iron has a dominant contribution to the Rosseland average in the same layers where large overabundances of Fe can be supported by the radiation field.

In stellar atmospheres, and especially magnetic atmospheres, the computation of radiative accelerations is much heavier to carry out than for internal layers where the diffusion approximation (Milne, 1927) may be applied safely. In optically thin cases, these computations require to solve in detail the radiation transfer equations along the line profiles in each depth of the atmosphere (including the Zeeman effect for magnetic cases Alecian \& Stift, 2004).

\footnotetext{
${ }^{2}$ Radiative accelerations of abundant elements vary as the inverse of the square root of the abundance. This is due to saturation of lines when Lorentz profiles (Alecian \& LeBlanc, 2000) are assumed. The final overabundance of iron should certainly be lower than that maximum value. The real abundance will result from the complex time dependent non-linear build-up of the iron stratification (Richer et al., 2000).
} 

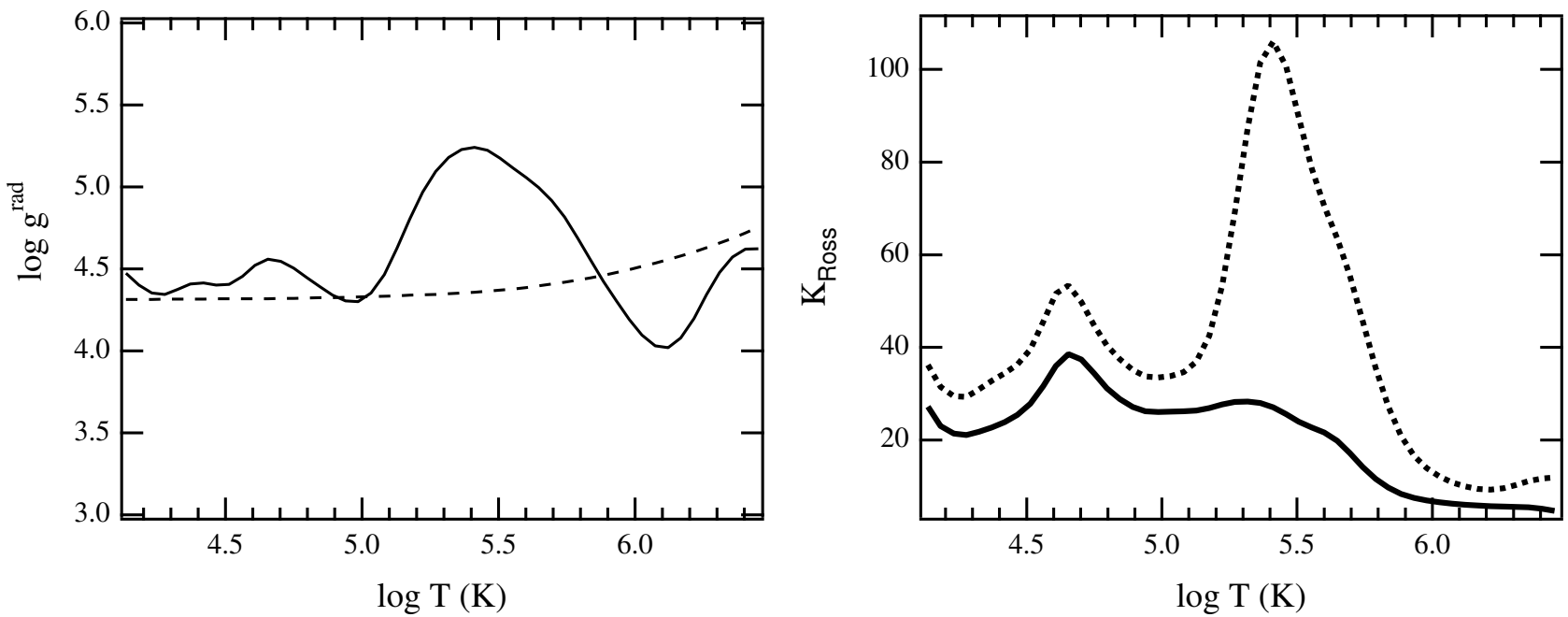

Figure 2. Left panel: radiative acceleration of Fe vs. layer's temperature (all in log). Dashed line is gravity. Right panel: Rosseland averaged opacity for solar (solid line) abundances and for 10 times the solar abundance of iron (dotted curve). The stellar model corresponds to a main sequence star with $T_{\text {eff }}=12000 \mathrm{~K}$ (from Alecian, 2007).

\section{Build-up of abundance inhomogeneities and diffusion time scales}

In stable medium, calculating the diffusion velocity of each element according to the depth is not enough in estimating what abundance may be found in a given stellar layer. In the 1970s, the first qualitative argument was to consider that elements that are strongly pushed up from deep layers to upper ones (positive velocity) may at least accumulate in the atmosphere. Indeed, some good correlations were found between elements with strong radiative acceleration and overabundances determined in the atmospheres of Ap stars (Michaud, 1970). Later on, more detailed calculations allowed in estimating for a given element, the maximum abundance that can be supported by the radiation field. This is equivalent to compute for each depth, for what abundance the radiative acceleration $^{3}$ has the same modulus as gravity. For such an abundance stratification, the diffusion velocity almost vanishes everywhere (and so also the particle flux). This is called the equilibrium solution.

\subsection{Build-up of abundance inhomogeneities}

Actually, to describe how abundances evolve according to the depth for an element $k$ with particles number density $n_{k}$, one must solve the time dependent continuity equation that may be written as:

$$
\partial_{t} n_{k}+\nabla\left[\mathrm{n}_{\mathrm{k}} \cdot\left(\mathbf{V}_{\mathrm{D}_{\mathrm{k}}}+\mathbf{V}_{\mathrm{M}}\right)\right]=0,
$$

where $\mathbf{V}_{D_{\mathrm{k}}}$ is the diffusion velocity specific to $k, \mathbf{V}_{\mathrm{M}}$ is the velocity of a macroscopic motion (the same for any $k$ ) like a wind, a large-scale circulation, etc. The equilibrium solution mentioned above is a particular stationary solution of this equation (assuming $\mathbf{V}_{\mathrm{M}}=0$ ).

In stellar interiors, this equation (3) is solved all along the stellar evolution (see for instance Deal et al., 2016, Michaud et al., 2011, Théado et al., 2009).

For atmospheres, almost all quantitative results for abundance stratifications published before 2018, give equilibrium solutions (for instance Alecian \& Stift, 2010, LeBlanc et al., 2009). Because these stratifications are simply solutions of Eq. 3 forcing $\partial_{t} n_{k}=0$ and $\mathbf{V}_{\mathbf{M}}=0$, there is no conservation of particle numbers. It corresponds just to the maximum abundance that can be supported by the radiation field in each layer. Actually, it is not trivial to know in which case, and for which element,

\footnotetext{
${ }^{3}$ Due to saturation effect on the line profiles, radiative acceleration decreases when element abundance increases.
} 

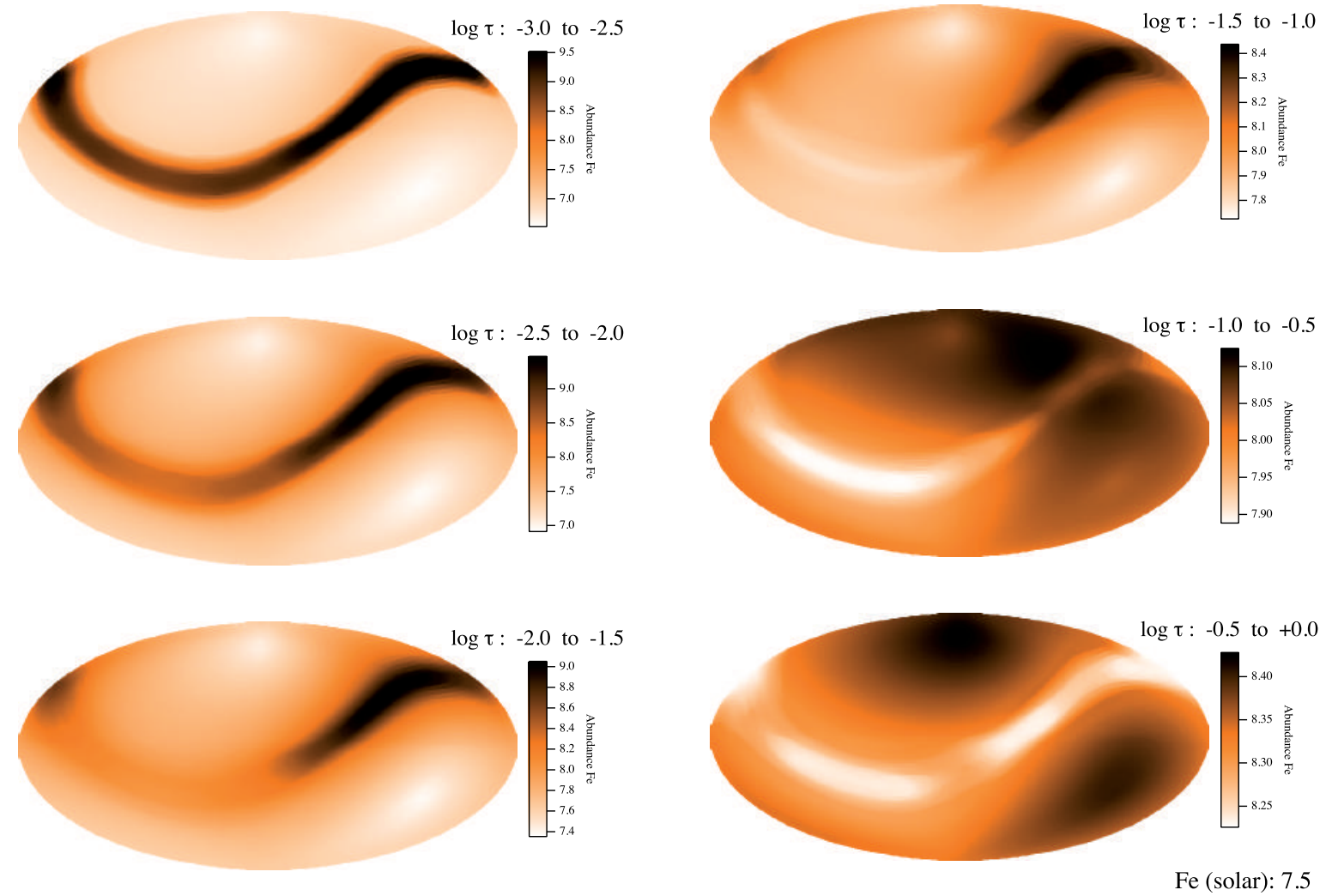

Figure 3. Tomographic view of the Fe abundance (Hammer equal-area projection) in a magnetic atmosphere (Alecian \& Stift, 2017). Six slabs corresponding to six contiguous optical depth ranges (indicated above each projection) are shown. The solar abundance we adopted for Fe is 7.5.

equilibrium solution corresponds to what happens in real atmosphere. However, because these calculations of equilibria do not need extensive numerical resources, they allow to put more efforts in other aspects of the modelling, as for instance computation of the 3D abundances distribution in magnetic atmospheres for which some interesting results have been obtained (Alecian \& Stift, 2017) as shown in Fig. 3.

A better approach, but numerically challenging and much more costly, is to solve the time dependent equation (with all the terms of Eq.3) from an initial time when abundances are homogeneous, and let them evolve. This was done first for atmospheres (with and without magnetic fields) by Alecian et al. (2011), but still assuming $\mathbf{V}_{\mathrm{M}}=0$. Stift \& Alecian (2016) has shown that solutions of the time-dependent equation often converge (after a few decades of physical evolution time) towards stationary solutions (constant particle flux throughout the atmosphere). But these simulations never converge towards equilibrium, at least for the cases without numerical instabilities. Moreover these stationary stratifications are very different from those assuming equilibrium. One therefore wonders to what extent equilibrium solutions can be appropriate in describing real atmospheres. This question is still open. On another hand, one cannot exclude that some of the instabilities experienced during these numerical simulations are physical (Alecian et al., 2011) and could concern precisely evolution toward equilibrium solutions. Recently, a new step in these studies have been overcome by Alecian \& Stift (2019) by introducing mass loss in that numerical simulations $\left(\mathbf{V}_{\mathrm{M}} \neq 0\right)$ for atmospheres.

\subsection{Time scales}

It is interesting at this point to discuss the time needed for abundances to stratify. A characteristic diffusion timescale may be defined as the time needed for particles to diffuse along a pressure scale height. This quantity, which depends on the particle type and on local plasma conditions, is estimated after the computation of the diffusion velocity for that type of particle. 
For atmospheres, typical atomic diffusion time scales are shown in Fig. 3.2 for $\mathrm{Fe}$ and $\mathrm{Hg}$ (from Alecian et al. 2011). One may notice that there is a large difference of the time scales of $\mathrm{Hg}$ compared to the one of Fe: mercury diffuses much faster than iron. This difference is mainly due to the fact that these timescales are computed at solar abundances: $\mathrm{Hg}$ has much lower abundance than Fe, and then has a stronger radiative acceleration. During the stratification process, the time scales will change according to the local abundances. Because the chemical stratifications build-up is strongly non-linear, the knowledge of these instantaneous time scales is not enough to estimate the time needed to form the observed concentrations of elements, but are helpful in estimating the efficiency of this transport process, especially if it has to be compared with macroscopic motions.

The timescales in Fig. 3.2 are strongly decreasing (several orders of magnitude) when one goes higher in the atmosphere (the higher the curves, the shorter the timescales). This is mainly due to the diffusion coefficient which varies as $n_{p}{ }^{-1}$ ( $n_{p}$ is the number density of protons). Larger is the diffusion coefficient, shorter is the time scale. For $\mathrm{Hg}$, one can see that the diffusion timescale at solar abundance is smaller than 1 month for $\log \tau<-3$. Therefore it seems possible that observed $\mathrm{Hg}$ overabundances in a $\mathrm{HgMn}$ stars could change significantly between two observations separated by a few years. Such a case was observed by Kochukhov et al. (2007). For deeper layers, the time scale continues to increase drastically, so much that it becomes larger than the age of the star (see an example in Alecian, 2009).

\section{Conclusion}

It is now well accepted in stellar community that the hypothesis of homogeneous abundances outside the stellar core for main-sequence stars was often wrong. Modelling stars with all processes involved in separating elements inside stellar medium is difficult since, atomic diffusion is a slow process and so, always confronted by several other mechanisms. However, a lot of theoretical/numerical works have been carried out since the 1970s, and this helped in much better understanding of elements distributions outside the core. For normal stars, discrepancies with standard models revealed by accurate asteroseismic observations are also better understood, since elements distribution change local opacities, which determine seismic behaviours.

Despite recent progress, atomic diffusion remains a difficult challenge for numerical modelling. It is not yet possible to reproduce theoretically the observed abundances of individual stars. However, theoretical models for atmospheres help in better explaining the strangeness of chemically peculiar stars: diversity inside a given group, stratification, horizontal inhomogeneity, dependence on magnetic fields, etc. Time-dependent simulation of atomic diffusion (including mass loss) appears to be very promising, and gives hope to be able to confront in a near future theoretical results in atmospheres with observations of CP stars. 


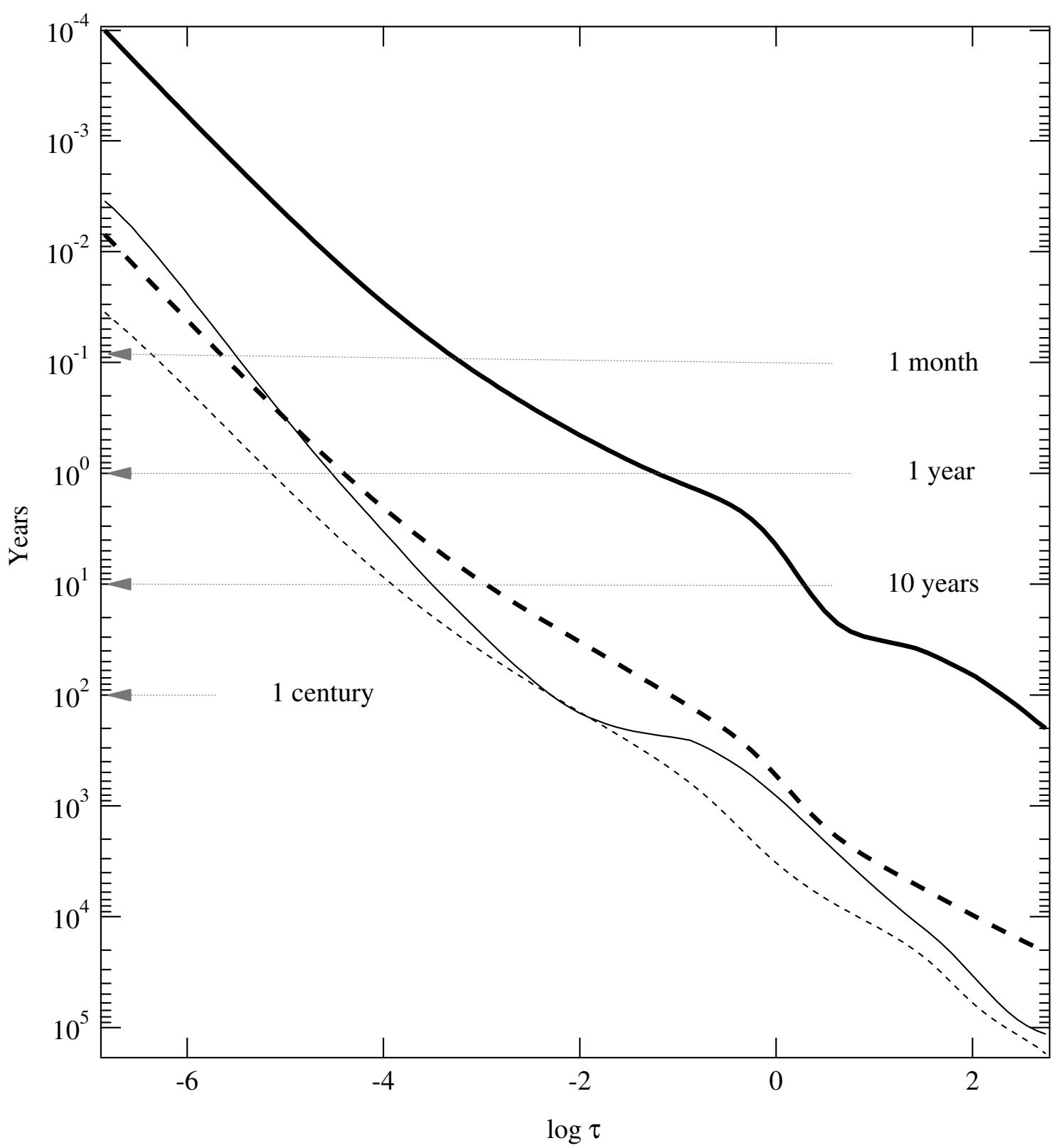

Figure 4. Diffusion time scales for Fe and $\mathrm{Hg}[\log ($ years $)$ vs. $\log ($ optical depth at $5000 \AA)]$ for a non-magnetic atmosphere with $T_{\text {eff }}=12000 \mathrm{~K}, \log g=4.0$. Solid lines correspond to the complete diffusion timescales, dashed lines to gravitational settling (diffusion without radiative acceleration). Heavy lines are for Hg, the others for Fe. (From Alecian et al. 2011). 


\section{Acknowledgements}

The ComBAO would like to the thank the dedicated researchers who are publishing with the ComBAO.

\section{References}

Alecian G., 2007, in C. W. Straka, Y. Lebreton, \& M. J. P. F. G. Monteiro ed., EAS Publications Series Vol. 26, EAS Publications Series. pp $37-48$

Alecian G., 2009, Communications in Asteroseismology, 158, 34

Alecian G., 2013, in Alecian G. L. E. R. O. V. G., ed., EAS Publications Series Vol. 63, EAS Publications Series. pp 219-226

Alecian G., 2014, in Guzik J. A., Chaplin W. J., Handler G., Pigulski A., eds, IAU Symposium Vol. 301, IAU Symposium. pp $185-191$

Alecian G., LeBlanc F., 2000, MNRAS, 319, 677

Alecian G., Stift M. J., 2004, A\&A, 416, 703

Alecian G., Stift M. J., 2010, A\&A, 516, A53+

Alecian G., Stift M. J., 2017, MNRAS, 468, 1023

Alecian G., Stift M. J., 2019, MNRAS, 482, 4519

Alecian G., Stift M. J., Dorfi E. A., 2011, MNRAS, 418, 986

Chapman S., Cowling T. G., 1970, The mathematical theory of non-uniform gases. an account of the kinetic theory of viscosity, thermal conduction and diffusion in gases

Deal M., Richard O., Vauclair S., 2016, A\&A, 589, A140

Ghazaryan S., Alecian G., Hakobyan A. A., 2018, MNRAS, 480, 2953

Ghazaryan S., Alecian G., Hakobyan A. A., 2019, arXiv e-prints, MNRAS, in press

Kochukhov O., Adelman S. J., Gulliver A. F., Piskunov N., 2007, Nature Physics, 3, 526

LeBlanc F., Monin D., Hui-Bon-Hoa A., Hauschildt P. H., 2009, A\&A, 495, 937

Michaud G., 1970, ApJ, 160, 641

Michaud G., Richer J., Richard O., 2011, A\&A, 529, A60

Michaud G., Alecian G., Richer J., 2015, Atomic Diffusion in Stars, Astronomy and Astrophysics Library, Springer International Publishing, Switzerland.

Milne E. A., 1927, MNRAS, 87, 697

Richer J., Michaud G., Turcotte S., 2000, ApJ, 529, 338

Ryabchikova T., 2005, in Alecian G., Richard O., Vauclair S., eds, EAS Publications Series Vol. 17, EAS Publications Series. pp $253-262$

Stift M. J., Alecian G., 2016, MNRAS, 457, 74

Théado S., Vauclair S., Alecian G., LeBlanc F., 2009, ApJ, 704, 1262

Thiam M., Leblanc F., Khalack V., Wade G. A., 2010, MNRAS, 405, 1384

Turcotte S., Richer J., Michaud G., Iglesias C. A., Rogers F. J., 1998, ApJ, 504, 539

Watson W. D., 1971, A\&A, 13, 263 